\title{
Patient Blood Management
}

\section{Weniger Transfusionen verbessern das Outcome}

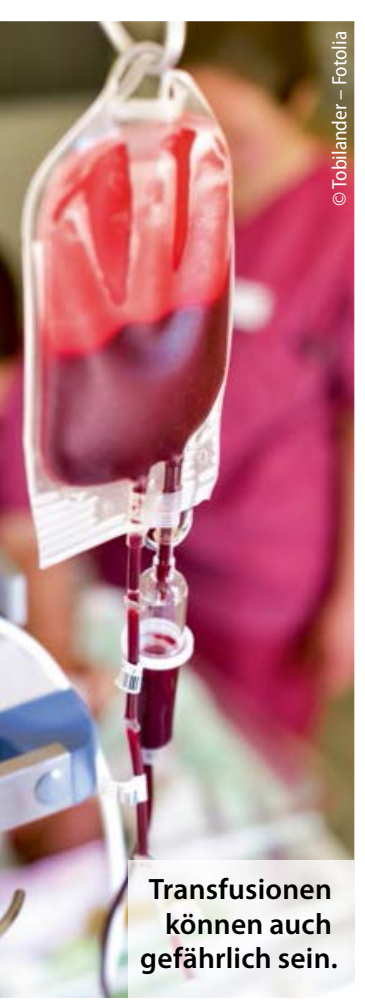

\begin{abstract}
_ Unter Patient Blood Management (PBM) versteht man ein evidenzbasiertes, multidisziplinäres, multimodales Behandlungskonzept für Blutungen und Operationen, bei dem die Optimierung und Bewahrung des patienteneigenen Blutvolumens im Mittelpunkt steht. Der Bedarf an Bluttransfusionen soll dadurch reduziert werden.
\end{abstract}

„Das Konzept beruht auf drei Säulen, nämlich der Optimierung des Erythrozytenvolumens durch Korrektur einer Anämie z. B. mit einem Eisenpräparat, der Mi- nimierung des Blutverlustes bei der Operation und der Vermeidung von Bluttransfusionen“, sagte Dr. Axel Hofmann, Laxenburg, Österreich. Jede Bluttransfusion sei mit einer Reihe von Komplikationen assoziiert, insbesondere einer Zunahme an nosokomialen Infektionen, aber auch ischämischer Ereignisse wie Myokardinfarkten. Darüber hinaus kann es $\mathrm{zu}$ einer transfusionsassoziierten Volumenüberladung oder einem Lungenversagen kommen. Durch eine Reduzierung allogener Erythrozytentransfusionen würden die Mortalität, die Morbidität und die Krankenhausverweildauer deutlich gesenkt, so Hofmann.

\section{ROTEM ${ }^{\circledast}$-Diagnostik unverzichtbar}

Einen wichtigen Beitrag zu dieser Strategie leistet die ROTEM ${ }^{\circ}$-Diagnostik. Diese Methodik, die auf dem Prinzip der Thrombelastometrie basiert, ermöglicht eine unmittelbare und genaue Einschät- zung des Gerinnungsstatus - eine unverzichtbare Voraussetzung für ein zielgerichtetes PBM. Die Analysen lassen sich patientennah auch in der Notaufnahme, im Operationssaal oder auf der Intensivstation durchführen. Das Verfahren liefert Informationen über eine Hyperfibrinolyse, das Ausmaß einer Verbrauchskoagulopathie sowie den Substitutionsbedarf von Fibrinogen, Gerinnungsfaktoren und Blutplättchen. Aufgrund der Vollautomatisierung sind keine Pipettier- oder Testvorbereitungsschritte notwendig.

Die konsequente Umsetzung von PBM führe laut Prof. Donat Spahn, Zürich, auch zu wesentlichen Einsparungen, die weit über die reduzierten Kosten für nicht verbrauchte Blutprodukte hinausgingen.

Red.

- Media Advisory Board: „Patient Blood Management und ROTEM : Die medizinischen, ökonomischen und analytischen Fakten"; München, Juni 2015 (Veranstalter: Tem)

\section{Mehr als moderne Medikamente}

\section{Der Patient mit Diabetes mellitus im Fokus}

— „Kommunikation ist nicht alles, aber ohne Kommunikation ist alles nichts" unter diesem Motto informierten unter Vorsitz von Prof. Norbert Hermanns und PD Dr. Bernhard Kulzer, Bad Mergentheim, Referenten im Rahmen des ersten Symposiums der Firma BerlinChemie über neue Konzepte zur Schulung und Information von Menschen mit Typ-1-Diabetes.

Erstmals wurde das neue PRIMASErstschulungsset für die Diabetespraxis vorgestellt. Inwieweit PRIMAS Einfluss auf die Praxis hat, zeigten die Ergebnisse der PRIMUM-Studie. Welchen Nutzen das TheraKey-Konzept für Arzt, Patient und Angehörige hat, wurde in einem sich anschließenden Vortrag erläutert. Mit dem Konzept erhalten Menschen mit Typ-1-Diabetes und ihren An- gehörigen die Möglichkeit, sich außerhalb der Sprechstunde über ihre Erkrankung zu informieren.

Im Symposium „Der multimorbide Typ-2-Diabetiker: ,Hand aufs Herz' - haben wir alles im Blick?" berichteten die Referenten über die Herausforderung von Begleiterkrankungen in der Therapie von Patienten mit Typ-2-Diabetes. Die nichtalkoholische Fettleber war Thema des Vortrages von Prof. Andreas Birkenfeld, Dresden. Warum kardiovaskuläre Erkrankungen und Nierenfunktionseinschränkungen ein Problem in der Diabetestherapie sind, erläuterte Dr. Axel Versen, Friedrichshafen.

\section{Das Mehr in der Diabetestherapie}

$\mathrm{Ob}$ Schulungsprogramme, Fortbildungsveranstaltungen oder Förderprei- se: Berlin-Chemie bietet neben Medikamenten unterschiedlichste unterstützende Maßnahmen zu einer besseren Versorgung von Menschen mit Diabetes an. Am Ausstellungsstand konnten sich Besucher über das Engagement des Unternehmens umfassend informieren, z.B. auch über das Kommunikationskonzept TheraKey".

Mit verschiedenen Materialien und Maßnahmen, wie dem TheraKey ${ }^{\circ}$ Onlineportal, der TheraKey'-MyTherapyApp zur Verbesserung der Adhärenz oder laienverständlichen Schaukarten, möchte das Unternehmen das Arzt-Patienten-Gespräch ganzheitlich unterstützen.

Red.

- Nach Informationen von Berlin-Chemie 